\title{
FORMAÇÃO DE PROFESSORES PARA A PROMOÇÃO DE PROJETOS DE INCLUSÃO DIGITAL SUSTENTÁVEIS
}

\author{
Magda Pischetola*
}

\section{Resumo}

Muitas iniciativas políticas dos últimos anos visam promover projetos de inserção das Tecnologias de Informação e Comunicação (TIC) no âmbito escolar, a partir do pressuposto que o acesso físico à ferramenta tecnológica por si só é fator de inclusão social e cultural. O presente artigo discute criticamente esta visão determinística, propondo concentrar a atenção nos elementos culturais, nas percepções individuais da tecnologia e no seu uso concreto no cotidiano da sala de aula. Para tal, o texto redefine o conceito de inclusão digital e apresenta algumas possibilidades a de trabalho nos encontros de formação de professores, focadas no desenvolvimento de competências e na atualização das práticas de ensino. O objetivo dessa proposta é construir um quadro de intervenção educacional que garanta a sustentabilidade de um projeto de inclusão digital, não só de um ponto de vista econômico, mas também social e cultural.

Palavras-chave: Formação. Inclusão digital. Mídias digitais. Sustentabilidade. Educação.

\section{TEACHERS' TRAINING FOR SUSTAINABLE DIGITAL INCLUSION PROJECTS}

\begin{abstract}
The implementation of digital devices represents one of the main educational trends in recent years, alongside the improvement in wireless connections potential. Many political initiatives have address development and innovation issues through projects of digital inclusion, often taking for granted that access to Information and Communication Technologies is the main factor to produce differences in social, cultural and economic outcomes. The paper goes beyond this deterministic vision, in favor of a broader concept of digital inclusion. The framework proposed is a model based on cultural needs analysis and subsequent promotion of didactics innovation, alongside the enhancement of competencies development, both from teachers and pupils. The main goal is to present a sustainable approach to digital inclusion, having as a focus methodological training for teachers.
\end{abstract}

Keywords: Training. Digital inclusion. Digital media. Sustainability. Education

\footnotetext{
* Doutorado em Educação. Professora da Universidade Católica do Sagrado Coração - Itália. E-mail: magda_pischetola@yahoo.it
} 


\section{Introdução}

Nos últimos anos, muitos projetos ao redor do mundo têm abordado o desenvolvimento humano e social por meio da introdução de Tecnologias de Informação e Comunicação (TIC) nas escolas, com o objetivo de gerar a inclusão digital (BONILLA E PRETTO, 2011; FANTIN, 2012; PISCHETOLA, 2011; WARSCHAUER, 2006). Uma grande parte destes projetos não teve continuidade após o primeiro/segundo ano da implementação, por não levar em conta alguns fatores-chave da integração da tecnologia, desde os aspectos sociais até a falta de assistência técnica. Neste sentido, falamos de insustentabilidade de um projeto (SCHUNK, 2004).

Segundo a Comissão Europeia, um projeto é sustentável se as soluções encontradas no curto prazo conseguem ter um resultado positivo também no médio e no longo prazo (COMMISSION DES COMMUNAUTÉS EUROPÉENNES, 1993). Isso pode acontecer, por exemplo, no caso em que os programas interpretem as necessidades das universidades, associações, ONGs, as estruturas públicas locais, com particular ênfase na transferência de know-how. Assim, fundar um projeto coerente e completo implica estabelecer uma rede de apoio entre as instituições e fazer uma previsão, já em fase de planejamento, dos possíveis fatores, complicações e problemas que irão influenciar as intervenções das fases subsequentes. Portanto, temos um projeto que funciona efetivamente quando temos a eficiência em termos de qualidade, flexibilidade e capacidade de adaptação às circunstâncias, confiabilidade, economia operacional e, sobretudo, sustentabilidade cultural, ou seja, radicação do projeto na realidade local (PISCHETOLA, 2011).

Para abrir o caminho para um projeto de inclusão digital na escola, primeiro é necessário considerar que

em qualquer contexto, as diferenças locais podem criar ou destruir as suas perspectivas: se tomarmos medidas inadequadas do ponto de vista econômico, tecnológico ou de gestão, se o programa não é adequado para as condições locais, [...] mesmo o melhor projeto está condenado ao fracasso (BLACK, 2002, pag. 138).

Com base nos elementos recolhidos em algumas pesquisas realizadas, ou em andamento (BONILLA E PRETTO, 2011; FANTIN E RIVOLTELLA, 2012; PISCHETOLA, 2011; WARSCHAUER, 2006), construiu-se um quadro de intervenção educacional que garanta a sustentabilidade de um projeto. A proposta deste artigo é que este quadro esteja centrado na formação de professores. 


\section{Sobre o conceito de inclusão digital}

A inclusão digital é foco de políticas públicas em todos os níveis da administração pública, bem como de ações de diversas instituições privadas, públicas e sem fins lucrativos. O discurso sobre inclusão digital tem gerado projetos de intervenção educativa baseada em distribuição de TIC, ampliação do acesso aos computadores, formação técnica dos professores (WARSCHAUER, 2006). Algumas dessas abordagens de inserção das TIC na escola parecem afirmar que uma nova tecnologia, pelo simples fato de chegar num contexto escolar, terá um efeito de inclusão digital. Esta visão é comumente conhecida como determinismo tecnológico (PEIXOTO, 2009).

Sem dúvida, dar acesso às tecnologias é uma condição técnica imprescindível e básica para qualquer projeto de inclusão digital, mas a nossa proposta é considerar a inclusão digital como verdadeira inclusão cidadã à cultura digital. Isso representa um desafio à ideia de que a inclusão digital seja apenas um problema econômico ou de infraestrutura, e abre o caminho a uma definição mais completa de inclusão digital como problema cultural (LEMOS, 2007; PISCHETOLA, 2011; RIVOLTELLA, 2003). Com a integração das TIC na didática, a escola pode assegurar um melhor uso das oportunidades da cultura digital para alcançar seus objetivos formativos.

Em vez de partir do pressuposto de que existe uma relação determinística entre tecnologia e inclusão, propõe-se então uma reflexão com base em três eixos:

1. O uso da tecnologia depende/faz parte da cultura, por isso é indispensável conhecer o contexto cultural onde o projeto vai atuar.

2. Há uma necessidade de questionar o pressuposto de que as TIC se inserem facilmente no âmbito escolar e aprofundar o discurso sobre as competências necessárias.

3. Finalmente, há um equívoco em muitos dos atuais debates sobre formação dos professores, entendida só como formação técnica. Para a sustentabilidade de um projeto, interessa-nos desenvolver novas práticas didáticas com a mídia, e não só competências técnicas.

Nos próximos parágrafos vamos aprofundar estes três aspectos.

\section{Analisar as necessidades de um contexto cultural}

Afirmamos que a inclusão digital é um processo cultural. Essa linha de raciocínio 
também nos leva a entender que o primeiro passo na concepção de um programa de inclusão digital corresponde o reconhecimento das necessidades específicas da realidade onde o programa será implementado. De fato, numa concepção coerente de uma intervenção para implementar as TIC em contexto educativo, é essencial ter em conta o potencial interesse dos destinatários a quem é dirigida, já que o interesse é um sintoma de uma necessidade (MASLOW, 1954). A primeira coisa a verificar seria, então, a percepção inicial da importância das TIC para os destinatários, pois o potencial das ferramentas tecnológicas só pode ser entendido se elas forem reconhecidas como sendo especialmente relevantes para o cotidiano (pessoal e profissional) das pessoas envolvidas. Um contexto que mostra a curiosidade e vontade de aprofundar pelos professores é, certamente, mais promissor do que um contexto onde há falta de motivação (FREIRE, 1977).

Com essa perspectiva em mente, reconhecemos que a criação de um canal de comunicação entre os promotores e os destinatários de um projeto é de fundamental importância, e deve ter em mente dois objetivos: por um lado gerar (ou amplificar) a curiosidade já existente pela tecnologia, por outro diminuir a eventual divinização ou demonização da tecnologia (RIVOLTELLA, 2003; MAYER, 2001). Pesquisas de campo (GOUROVA, 2001; WARSCHAUER, 2006) constatam que as percepções dos beneficiários em relação às possíveis mudanças na escola e na comunidade como resultado de projetos de inclusão digital são muitas vezes distorcidas, tanto em termos negativos quanto em termos positivos. O objetivo dos primeiros encontros de formação com os professores não é corrigir esses pontos de vista, a maioria causada por falta de conhecimento e não-utilização de ferramentas tecnológicas, mas sim fornecer uma orientação clara sobre a proposta do projeto. Um aspecto de maior consideração, por exemplo, é a atenção para o tempo de implementação das TIC na escola: deve ficar claro que, também no caso em que as tecnologias sejam adotadas com sucesso dentro da escola, dificilmente serão capazes de produzir efeitos imediatos ou em curto prazo na geração de novas capacidades ou aprendizagens.

\section{Promover as competências de alunos e professores}

Diversas pesquisas salientam que a inovação depende das variáveis sociais e culturais e, portanto, é fundamental adotar uma atitude crítica para entender a tecnologia como uma área formativa (GEE E HAYES, 2011; WARSCHAUER, 2006). Nesta perspectiva, afirmamos a importância de uma formação que tenha em conta o contexto sócio cultural e proporcione o desenvolvimento das habilidades dos alunos e professores. O uso de tecnologia 
em primeiro lugar requer muita consciência da complexidade de gerenciar não apenas novas ferramentas, mas as condições de aprendizagem diferentes (GOUROVA, 2001).

O primeiro problema para o professor, diz Varisco (2002), é gerenciar ambientes que parecem “caóticos”:

É óbvio que envolver os alunos na seleção de atividades a serem realizadas, respeitando ao mesmo tempo as regras e os tempos da sala de aula, significa que o ambiente de ensino não é completamente determinado pelo professor, mas, pelo contrário, deixa um certo nível de incerteza e de falta de controle, o que coloca o professor em um constante estado de impermanência e cautela (VARISCO, 2002, pag. 158).

Isto é especialmente verdadeiro no caso da introdução de uma tecnologia: 1 (um) laptop por aluno, que requer o uso responsável de cada um, especialmente quando se trata de grandes classes e crianças pré-adolescentes (PISCHETOLA, 2011). O professor deve ganhar confiança no uso tecnologia, enquanto ao mesmo tempo deve acompanhar e orientar o desenvolvimento de competências digitais dos alunos. As formas de trabalho com esse tipo de tecnologia necessitam de dinâmicas colaborativas e negociações contínuas, com o resultado que os benefícios destas situações de ensino em geral parecem mais teóricos do que reais. Contudo, trata-se de dificuldades iniciais. Uma vez que acabe a fase de implementação do projeto, ou seja, quando os alunos ganharam autonomia suficiente e o professor aprendeu a gerenciar as atividades em sala de aula, a tecnologia vai, sim, ser um facilitador do ensino, permitindo uma considerável economia de tempo para completar as tarefas, junto à participação ativa dos alunos. Além disso, se os alunos se tornam mais independentes na atividade de aprendizagem, o professor pode propor intervenções mais específicas e personalizadas.

É claro que a gestão e organização das atividades com a mídia também afeta a abordagem cultural do ensino e da aprendizagem (ARDIZZONE E RIVOLTELLA, 2008). O método de ensino é, sem dúvida, mais eficaz se é flexível e aberto para o planejamento conjunto com os alunos, a fim de rapidamente eliminar a fase inicial de implementação de um projeto (VARISCO, 2002). Para que o projeto seja bem sucedido, a formação de professores deve, portanto, concentrar-se em uma abordagem de ensino aberta ao contínuo replanejamento.

Além disso, a integração da tecnologia na educação requer uma revisão de linguagens, enquanto as novas mídias pressupõem uma habilidade metalinguística e uma habilidade metacognitiva dos alunos na leitura e na composição de textos escritos e multimídia 
(FANTIN E RIVOLTELLA, 2012; GEE E HAYES, 2011; JEWITT, 2006). Este último aspecto implica que não apenas o estudante, mas também o professor possa adquirir estas habilidades e depois seja capaz de aplicá-las ao seu trabalho por meio de estratégias de investigação possíveis.

Para alcançar bons resultados, o desafio final para as habilidades do professor é fundar o seu método não apenas na organização funcional do conteúdo, mas também na construção de uma alfabetização flexível, que consiste na capacidade de reformular constantemente a informação adquirida (LEASK, 2001; SENGE, 2006).

Desta forma, obtemos um modelo que se aproxima ao ensinar "transformador" esperado por Gardner (1993), que dá ao professor a tarefa de evocar as qualidades ocultas dos alunos, em vez de exigir, como o ensino que o autor define “ritualizado", a imitação do professor pelo aluno.

Para resumir, a atenção do formador deve concentrar-se nas competências dos professores e dos alunos, identificadas como na figura a seguir.
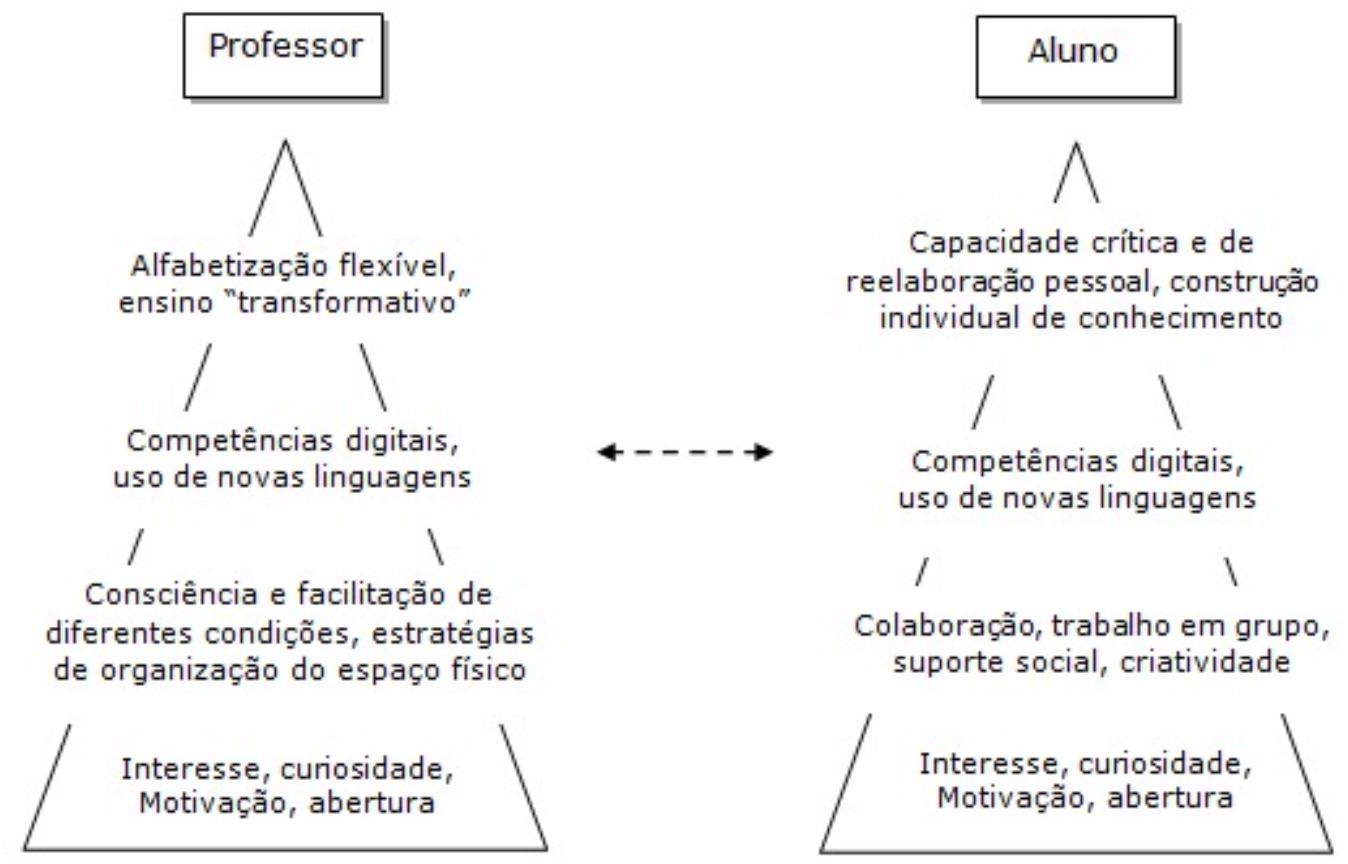

Fig. 1 - Construção de competências de professores e alunos (PISCHETOLA, 2011)

A aquisição de competências, que é o propósito essencial de um projeto, é o resultado de encontros de formação predispostos ao desenvolvimento do capital humano e do capital social na escola (PISCHETOLA, 2011). Um elemento-chave da reforma educacional é uma formação de professores que tenha como objetivo a articulação da introdução das TIC no 
currículo e a revisão das práticas de ensino, a fim de ajudar os professores no desenvolvimento das habilidades necessárias para lidar com a gestão de novos ambientes de aprendizagem.

\section{Rever as práticas didáticas para uma alfabetização digital significativa}

Um último aspecto importante na implementação de um projeto de inclusão digital é não superestimar a facilidade com que as tecnologias podem integrar-se às realidades existentes. De modo geral, na presença de instrumentos de trabalho diferentes, também temos que repensar as estratégias de ensino e aprendizagem. Entretanto, enquanto não há geralmente dúvida de que as novas tecnologias são mais que ferramentas essenciais para o desenvolvimento e inclusão, pois já são parte da própria cultura, um contraponto é que desenvolver novas metodologias didáticas com a integração das TIC ainda traz dificuldades para alguns professores.

Nessa perspectiva, sugerimos que se adote na formação o princípio da "utilização estratégica” das TIC, apresentado em 2004 pela UNESCO. Nessa visão, o uso da tecnologia é baseado principalmente na compreensão das diferentes ferramentas disponíveis (tipos de software, banco de dados, ferramentas de rede, etc.), e a capacidade do professor de determinar, de acordo com as necessidades e os recursos disponíveis, qual ferramenta utilizar em cada situação didática (UNESCO, 2004, pag. 26). Para fins de sustentabilidade cultural de um projeto, precisa-se de uma abordagem que combine o uso de laptops/tablets com outras mídias mais tradicionais (jornal, rádio, televisão), em relação aos tempos e formas de alcançar os objetivos que o professor queira alcançar.

Do ponto de vista das práticas de ensino com o uso da tecnologia, parecem ter sucesso as abordagens de aprendizagem por descoberta, que colocam o professor em posição de guia no processo de aprendizagem dos alunos (PISCHETOLA, 2011). O pensamento de Montessori acaba por ser mais atual do que nunca com o uso da tecnologia na educação: o foco está no processo e não no conteúdo, o fracasso é importante, o retorno do professor, sempre necessário, a compreensão pela criança mais profunda (MONTESSORI, 1970).

No entanto, reconhecemos que criar um ambiente de aprendizagem seguindo esta abordagem é muito mais difícil do que projetar uma série de intervenções pedagógicas tradicionalmente entendidas (ARDIZZONE E RIVOLTELLA, 2008). Por isso é tão importante que o professor tenha as competências para lidar com as situações, adaptando métodos e instrumentos a formas diferentes de conduzir o procedimento educativo. 


\section{Conclusões}

A formação de professores é um componente decisivo para a mudança na cultura escolar e nas concepções de realidade que determinam as modalidades do ensino. No entanto, a maioria dos projetos de intervenção educativa baseada em TIC não prevê suficiente atenção à formação, não investe, como deveria, em formadores especializados, limitando-se na maioria das vezes a uma formação técnica sobre o uso das ferramentas tecnológicas (WARSCHAUER, 2006). Isso acontece porque as soluções técnicas respondem às expectativas dos professores em curto prazo e parecem reduzir a necessidade de novas formações na escola. No entanto, a cultura centrada no professor é preservada, ou mesmo reforçada, assim como as práticas didáticas tradicionais. Na verdade, o que se propõe aqui é encarar as tecnologias como elementos de mudança e de inclusão social. Para que isso aconteça, é necessário pensar numa formação adequada, adaptada ao contexto cultural e focada no desenvolvimento das competências de alunos e professores, a partir dos interesses e das motivações individuais de cada um. Para que prevaleça o confronto positivo entre professor e aluno, é essencial em todas as fases de formação captar e incentivar a motivação dos professores, enfatizando a possibilidade de se aproximar aos poucos dos objetivos pedagógicos prefixados.

Disso decorre que o foco das formações seja o uso estratégico das TIC no cotidiano escolar, porque, como comentamos, na ausência desse uso estratégico, oferece-se pouca ajuda para o processo de desenvolvimento de uma comunidade escolar.

Não podemos esquecer que o objetivo fundamental da inclusão digital não é simplesmente reduzir o abismo digital entre tecnologia e infraestrutura, mas através do uso da tecnologia desencadear um círculo virtuoso de mudança social. Um projeto de inclusão digital deve ser capaz de oferecer uma oportunidade decisiva para reconfigurar a organização existente, respeitando os padrões culturais locais e tendo em mente o risco de distorcê-los, como resultado de um modelo aplicado sem considerar as características específicas do contexto.

Em conclusão, é papel da formação acompanhar os professores na experiência com a tecnologia, a fim de gerar atenção positiva para a partilha de novos conhecimentos. De fato, a formação pode mudar a percepção de tecnologia e criar certo entusiasmo na sua utilização. Deve, sobretudo, ser capaz de ativar reflexões pedagógicas e abrir novos horizontes culturais, que incluam a provisão para as práticas pedagógicas em constante mudança. 


\section{Referências}

ARDIZZONE, P., RIVOLTELLA, P. C. Media e tecnologie per la didattica. Milano: Vita \& Pensiero, 2008.

BLACK, M. The no-nonsense guide to international development. Oxford: New Internationalist, 2002.

COMMISSION DES COMMUNAUTES EUROPEENNES. Gestion du cycle de projet. Approche intégrée et cadre logique. Bruxelles: CCE, 1993.

FANTIN, M., RIVOLTELLA, P. C. Cultura digital e formação de professores: usos da mídia, práticas culturais e desafios educativos. In: Cultura digital e escolar: pesquisa e formação. Campinas: Papirus, 2012 (pp. 95-146).

FREIRE, P. Pedagogia da Autonomia: Saberes necessários à prática educativa. Lisboa: Edições BASE, 1977.

GARDNER, H. The Unschooled Mind: How Children Think and How Schools Should Teach. London: Fontana Press, 1993.

GEE, J. P., HAYES, E. Language and learning in the digital age. New York: Routledge, 2011.

GOUROVA, E. et al. The Digital Divide - A research perspective. European Commission: Joint Research Centre, 2001.

JEWITT, C. Technology, Literacy and Learning. London and New York: Routledge, 2006.

JONASSEN, D. H. Thinking technology, toward a constructivistic design model. Educational technology, n. XXXIV, April 1994(pp. 34-37).

LEASK, M. Issues in teaching using ICT. London and New York: Routledge Falmer, 2001.

LEMOS, A. (Ed.). Cidade digital: portais, inclusão e redes no Brasil. Salvador: Edufba, 2007.

MASLOW, A. H. Motivation and personality. New York: Harper \& Brothers, 1954.

MAYER, R. E. Multimedia learning. New York: Cambridge University Press, 2001.

MONTESSORI, M. Come educare il potenziale umano. Milano: Garzanti, 1970.

PEIXOTO, J. Tecnologia na Educação: uma questão de transformação ou de formação? In: CECILIO, S., FALCONE GARCIA D. M. (Eds.). Formação e profissão docente em tempos digitais. Campinas: Alínea, 2009.

PISCHETOLA, M. Educazione e divario digitale. Idee per il capacity building. Milano: Unicopli, 2011. 
RIVOLTELLA, P.C. Costruttivismo e pragmatica della comunicazione on line. Socialità e didattica in Internet. Trento: Erickson, 2003.

SENGE, P. M. The Fifth Discipline: The Art \& Practice of The Learning Organisation. London: Random House, 2006.

SCHUNK, J. The Chaos Theory Applied to International Co-operation. A Proposal to Go Beyond the Linear Logic. BeraterInnen News, n. 1, 2004.

UNESCO. ICT innovations for Poverty Reduction. New Delhi: UNESCO, 2004.

VARISCO, B. Costruttivismo socio-culturale: genesi filosofiche, sviluppi psico-pedagogici, applicazioni didattiche. Roma: Carocci, 2002.

WARSCHAUER, M. Tecnologia e inclusão social. São Paulo: Editora Senac, 2006.

Recebido em: junho de 2012 Aprovado em: novembro de 2012 